\title{
PhysioSkin: Rapid Fabrication of Skin-Conformal Physiological Interfaces
}

\author{
Aditya Shekhar Nittala ${ }^{1} \quad$ Arshad Khan $^{1,2} \quad$ Klaus Kruttwig $^{2}$ Tobias Kraus $^{2} \quad$ Jürgen Steimle $^{1}$ \\ ${ }^{1}$ Saarland University, Saarland Informatics Campus, Saarbrücken, Germany \\ ${ }^{2}$ INM-Leibniz Institute for New Materials, Saarbrücken, Germany \\ \{nittala, khan, steimle\}@cs.uni-saarland.de, \{klaus.kruttwig, tobias.kraus\}@leibniz-inm.de
}

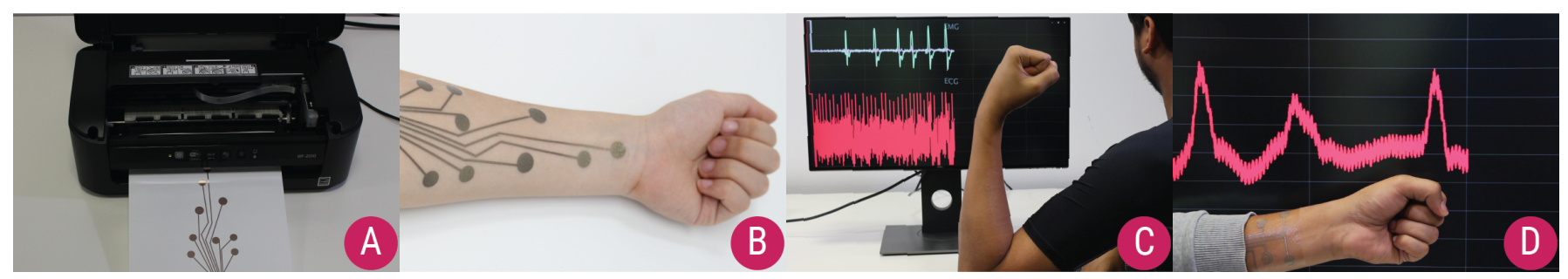

Figure 1. (a) PhysioSkin enables digital fabrication of custom electro-physiological sensing patches for monitoring EMG, ECG and EDA. (b) A custom made skin-conformal sensor. (c) A fitness tracking sportswear tracks heart rate and muscle movements. (d) Raw signal of the heart rate recorded from a temporary tattoo.

\section{ABSTRACT}

Advances in rapid prototyping platforms have made physiological sensing accessible to a wide audience. However, off-the-shelf electrodes commonly used for capturing biosignals are typically thick, non-conformal and do not support customization. We present PhysioSkin, a rapid, do-it-yourself prototyping method for fabricating custom multi-modal physiological sensors, using commercial materials and a commodity desktop inkjet printer. It realizes ultrathin skin-conformal patches $(\sim 1 \mu \mathrm{m})$ and interactive textiles that capture sEMG, EDA and ECG signals. It further supports fabricating devices with custom levels of thickness and stretchability. We present detailed fabrication explorations on multiple substrate materials, functional inks and skin adhesive materials. Informed from the literature, we also provide design recommendations for each of the modalities. Evaluation results show that the sensor patches achieve a high signal-to-noise ratio. Example applications demonstrate the functionality and versatility of our approach for prototyping a next generation of physiological devices that intimately couple with the human body.

\section{Author Keywords}

Physiological sensing; Fabrication; Rapid Prototyping; Ink-jet Printing; Wearable Devices; Electronic Skin; E-textile

\section{CCS Concepts}

-Human-centered computing $\rightarrow$ Interaction devices; Ubiquitous computing; Interaction techniques;

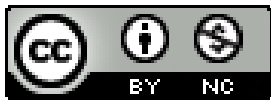

This work is licensed under a Creative Commons Attribution-NonCommercial International 4.0 License.

CHI'20, April 25-30, 2020, Honolulu, HI, USA

(C) 2020 Copyright held by the owner/author(s).

ACM ISBN 978-1-4503-6708-0/20/04. .

DOI: https : //doi .org/10.1145/3313831.3376366

\section{INTRODUCTION}

Physiological sensors are recently receiving increasing attention in the broad field of computing. While long used in areas related to health and rehabilitation [51], we are now witnessing an impressive array of new applications in interactive computing $[25,16,9]$. For instance, surface electromyography (sEMG) allows for detecting gestural input using unobtrusive wearable hardware [60]. Continuous monitoring of electrocardiogram (ECG) signals informs athletes about their performance [2] and monitoring of electrodermal activity (EDA) enhances computer-mediated emotional communication [58, $8,25,9]$. In parallel, accessible hardware platforms and toolkits make it easier than ever to implement interactive systems that include physiological sensing $[62,1]$.

Despite these advances, designers seeking to develop new applications are confronted with serious restrictions at the level of the computer-body interface: commercial gel-based electrodes are non-conformal, problematic at body locations that deform, and neither ergonomic nor aesthetic to wear during everyday activities. The materials community has contributed a number of devices that are ultra-thin and can sense multiple physiological modalities [21, 37]. However, these devices require complex fabrication processes and advanced lab equipment, which are typically inaccessible outside of specialized labs. They also require expertise and domain knowledge in multiple disciplines (materials science, bio-medical engineering) which can make if even harder for designers, practitioners and makers in realizing custom physiological sensing solutions.

To address this problem, we present PhysioSkin. We demonstrate that established digital fabrication techniques support printing customized electro-physiological sensor patches with advanced material properties that allow for accurately capturing sEMG, ECG and EDA signals. These patches readily work with off-the-shelf commodity physiological sensing 
toolkits [63, 67, 62], eliminating the need for building custom PCBs, and offering a rapid end-to-end pipeline for electrophysiological sensing. Our main contributions are:

1. We show that the digital fabrication of skin-conformal physiological sensing patches with advanced material properties is possible within 5-20 minutes, using a desktop inkjet printer and simple lab equipment. Through a systematic exploration of materials, functional inks, and skin adhesive materials, we present multiple fabrication approaches for sensors of customized thickness, stretchability, durability, and reusability. These realize sensors integrated in ultra-thin temporary tattoos $(\sim 1 \mu \mathrm{m})$, in stretchable TPU and PDMS materials, and in textiles. Our sensors contain dry electrodes and are orders of magnitude thinner than current off-the-shelf gel-based electrodes.

2. Technical evaluation results demonstrate that sensors fabricated using these techniques achieve high a signal-to-noise ratio for EMG and ECG signals and a high Pearson correlation coefficient(with respect to commercial gel-based electrodes) for EDA signals.

3. We show how sensing of multiple electro-physiological modalities (sEMG, ECG and EDA) can be integrated in a single patch. We furthermore demonstrate how to integrate electro-physiological sensing with user interface controls for touch input.

4. Informed from the literature in bio-medical engineering, we compile coherent design recommendations for the design of electrodes for each of the modality to pick up high-quality signals.

5. We demonstrate the practical feasibility and versatility of our approach by implementing three example applications: a textile vest for fitness tracking, a temporary tattoo for heart rate monitoring, and a PDMS-based patch for arousal logging in virtual reality environments.

\section{RELATED WORK}

\section{Physiological Sensing}

Design and fabrication of sensors for physiological monitoring has been subject of intense research in the materials and biomedical engineering communities. Thin epidermal devices have been presented that monitor various physiological signals such as ECG [77], EMG [21, 77], pulse oximetry [37], sweat and thermal characterization [32, 71]. Alternatively, textile sensors have been extensively explored as well [14], including for ECG [75], EMG [22], and EEG [40]. These solutions commonly require high-end tools and complex processes that are not compatible with the requirements of rapid prototyping.

The HCI community has explored using bio-signals for interaction. This involved the use of EMG signals for gesture recognition $[59,60,5]$. Various other bio-signals such as electro-dermal activity, heart-rate activity, and electroocculography have also been explored $[9,25]$. Other work investigated advanced device form factors for capturing bio-signals, for instance in shorts [13], in spectacles [17, 31] or through epidermal robots that move on skin [16]. This work, however, did not investigate rapid prototyping.

\section{Skin-Conformal Devices for Interaction}

Recent advances have enabled interactive devices for use on the skin that are thin, flexible and stretchable [72]. Ultra-thin devices based on temporary tattoos have been demonstrated that enable touch input and various forms of visual output [39, 34, 73, 47, 64]. More recent work has demonstrated the feasibility of feel-through tactile output using a temporary tattoo [74]. It has also been shown that on-skin devices can be fabricated to be completely untethered and easily removed and reapplied [41, 46].

\section{Rapid Fabrication of Flexible Circuits and Sensors}

To prototype custom flexible circuits and sensors in simple lab environments, researchers experimented with copper tape [53, 61] and conductive paint [42]. To enhance speed and resolution, conductive traces can be printed on substrates using an off-the-shelf inkjet printer filled with silver nanoparticle ink [35]. Printem [12] enables flexible PCBs with standard office printers and inks. Other rapid and do-it-yourself fabrication techniques have been contributed for various applications such as electronic circuits [70], paper circuits [54], textiles [49, 50], self-contained silicone devices [45], stretchable circuits [26], or cuttable sensors [48, 18]. More recent work has introduced printing of soft circuits on a wide variety of materials with a commodity inkjet printer [36], with a preliminary investigation of a skin-bound EDA sensor. However, digital fabrication of custom physiological sensors has been little explored. Commercial gel-based electrodes or metallic dry electrodes remain the de-facto standard in the $\mathrm{HCI}$ community for acquiring physiological signals.

\section{RECOMMENDATIONS FOR DIGITAL DESIGN}

The electro-physiological sensors investigated in this paper work by capturing electrical biosignals with electrodes on the human skin. A prerequisite for capturing high-quality biosignals is to place electrodes at carefully chosen locations. Our approach allows the designer to define these in a digital design, made in any 2D vector graphics application. In contrast to manually placing electrodes on the body, digital design offers both precise control and replicability.

Surface electromyography (sEMG) records muscle activity by reading the electrical potential generated by muscle cells, using two electrodes per muscle and an additional reference electrode. Electrocardiography (ECG) record the electrical activity of the heart which, amongst others, allows to identify heart rate. While it commonly involves 12 electrodes, a smaller number ( 3 in our implementation) is viable. Electrodermal activity (EDA) captures skin conductance, which varies with the state of sweat glands, and uses at least two electrodes.

Here we present a set of coherent design guidelines for the electrode design that we have compiled from the body of literature. Critical design choices relate to the size, location and arrangement of electrodes:

\section{Electrode Size}

The contact area of the electrode influences the quality of the signal. For EMG signal acquisition, the electrodes should have a minimum surface area of $50 \mathrm{~mm}^{2}$ and a diameter of less than $10 \mathrm{~mm}[29,78,44]$. For ECG, most prior research has typically 
designed electrodes in the range of 5-10 mm diameter [55, 56, 57]. For EDA signal acquisition, the recommended surface area is $1.0 \mathrm{~cm}^{2}$ [23]. We therefore select our electrodes sizes to be $10 \mathrm{~mm}$ diameter for EMG and ECG, and $12 \mathrm{~mm}$ diameter $\left(\sim 1.08 \mathrm{~cm}^{2}\right.$ area) for EDA electrodes.

\section{Location of Electrodes}

For EMG signals, the electrodes need to be placed on the muscle whose movement is to be captured.

For ECG measurements, electrodes are typically placed using the standard 12-electrode placement [33] or based on the Einthoven's triangle arrangement [19]. However, alternate placement strategies near both wrists and the forearm have also been suggested $[15,76,20]$. Our approach is based on prior work which designed 3-electrode ECG devices on the forearm $[76,28,3,20]$. This involves placing two electrodes on the forearm and the third electrode away from these measurement electrodes.

Since EDA electrodes measure the activity of sweat glands, they should be placed at locations that have a high density of sweat glands. The typical recommended locations are fingertips, palm (thenar and hypo-thenar eminence), foot sole and forehead $[23,11]$. However, prior work has also investigated the EDA response at various other locations on the body [69], which suggests that other locations such as forearm and wrist can deliver satisfactory performance, too.

\section{Inter-Electrode Distance}

The distance between the measuring electrodes plays a vital role in the signal acquisition. For EMG, the two measuring electrodes should be placed along the direction of the muscle. Their recommended distance depends on how deep the muscle is present beneath the skin. For muscles present on the surface, the recommended inter-electrode distance is $25 \mathrm{~mm}$; for deeper muscles, the distance is $40-50 \mathrm{~mm}$ [78, 7, 43]. For ECG measurements, we used inter-electrode distances from prior work [76], where the electrodes were placed around the arm with distance interval of $3 \mathrm{~cm}$. For EDA measurements an interelectrode distance of 5-6 cm has been successfully used in the previous literature $[69,6]$.

\section{FABRICATION}

The unique requirement for the fabrication of electrophysiological sensors is the need for low-impedance skincontacting electrodes. This is in contrast with prior work which contributed on-skin touch sensors [34, 47]. This adds challenges, most centrally at the level of electrode materials and skin adhesives, requiring different fabrication strategies.

Commercial solutions typically use electrodes covered by conductive wet gel to improve the electrical contact; however this makes the practical handling difficult and increase a device's thickness. Our solution uses more practical dry electrodes that we print in custom arrangements using conductive silver ink. The conformal nature of the substrates coupled with ultra-thin conductive traces ensures that the electrodes can successfully capture various bio-signals.

Leveraging on the ease and rapidity of inkjet printing, our fabrication approach builds on printing conductive traces with

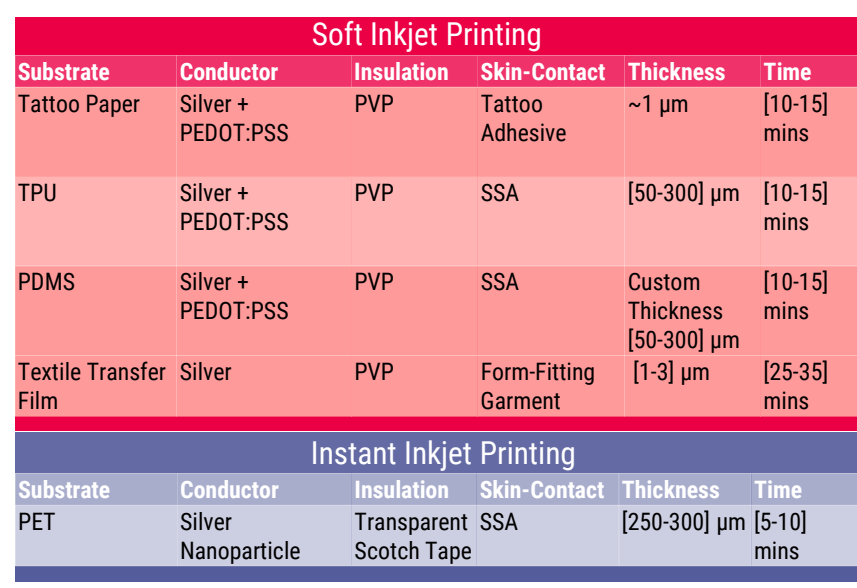

Figure 2. Overview of fabrication options with Soft inkjet and Instant inkjet printing.

a desktop inkjet printer, as previously presented by Kawahara et al. [35] and Khan et al. [36]. We contribute a systematic exploration of substrate materials, insulation mechanisms and adhesion schemes, demonstrating for the first time that desktop inkjet fabrication can realize sensors for various electrophysiological modalities using various materials. This opens up a design space of customized levels of device thickness, elasticity, robustness and fabrication speed. Figure 2 shows a comparative overview of all substrates and the associated compatible materials. We now present these different options.

Ultra-thin temporary tattoo sensor: Ultra-thin devices $(\sim 3-$ $4 \mu \mathrm{m})$ are realized by printing on commercial tattoo decal paper (SUNNYSCOPA, Printable Temporary Tattoo Paper for Laser Printer). Using the technique presented by Khan et al. [36], a layer with electrodes and connecting traces is printed using silver nano-particle ink and heat cured. Optionally, three layers of PEDOT:PSS conductive polymer using the same design can be printed first to enhance the mechanical robustness of the brittle metallic layer. Silver traces, but not electrodes, are then insulated by printing 5 layers of PVP (Polyvinylphenol, $\mathrm{Mw}=11,000 \mathrm{~g} / \mathrm{mol}$ ) on top. The layers are thermally cured, as indicated in [36]. A sheet of skin adhesive film (SUNNYSCOPA) is laser cut to leave electrode locations uncovered and then bonded onto the printed tattoo sheet. The sandwich can then be transferred onto skin.

Stretchable re-usable sensor using TPU or PDMS: While a tattoo device offers prime skin compatibility, it only supports one-time use. By using thicker elastic materials, superior robustness can be achieved while allowing for removing and reapplying the device. TPU (thermoplastic polyurethane) substrate $(\sim 50 \mu \mathrm{m}$ thick, $6.5 \mathrm{MPa})$ has high elasticity. Using the technique from [36], we print silver nano-particle ink on TPU with added 5 layers of PVP providing the insulation. The patch can be bonded to skin using skin adhesive film. Alternatively, one can use Soft Skin Adhesive (SSA) (MG-7-1010, Dow Corning), offering the benefit of applying and re-applying the patches multiple times.

Alternatively, PDMS (Sylgard 184, Dow Corning) offers similar mechanical properties. It is a substrate which has been extensively used for developing a wide range of epidermal devices, across disciplines. It offers great skin compatibility 


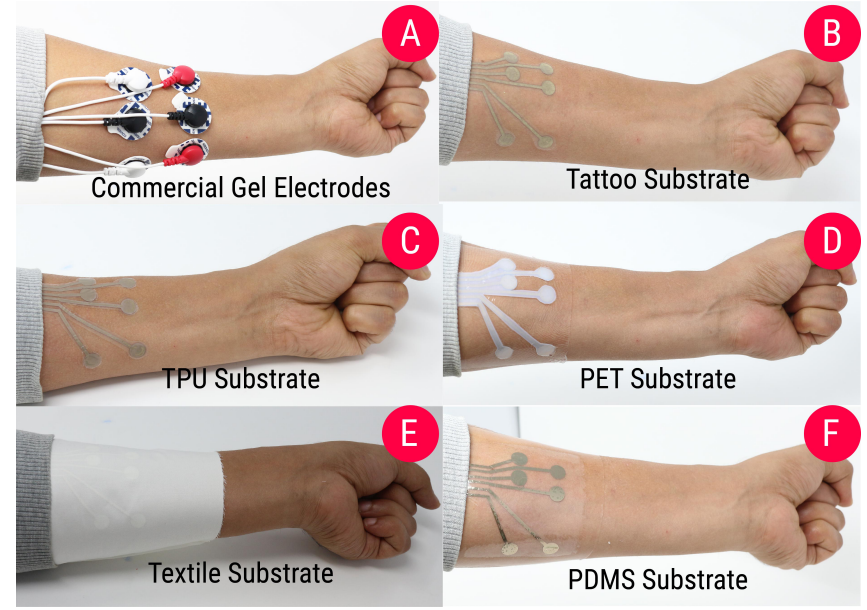

Figure 3. Conformal skin contact made by the electrodes fabricated on all the substrates.

and can be commercially acquired or self-fabricated in custom thickness and stretchability [45]. We cast a custom PDMS film ( $\sim 40 \mu \mathrm{m}$ thick, $\sim 2.7 \mathrm{MPa})$ using a doctor blade. Similar to TPU, the designs can be printed with conductive inks and applied on to the skin. However, as PDMS is hydrophobic a plasma treatment is required before printing on the substrate. For bonding to skin, we used a layer of SSA as a border dressing. PVP is used for insulation.

Textile-integrated sensor: We demonstrate that printing can realize functional skin-contacting electrodes that are seamlessly integrated on a textile. Informed by [36], we use commercial textile transfer film (SKULLPAPER, Premium Textile Transfer Film) and print electrodes and electrical connections using silver. The electrical connections are insulated by printing 5-6 layers of PVP. We create a negative mask of the design and laser-cut the textile transfer film after printing. This ensures that only the electrodes and traces are transferred onto the textile, leaving all other parts of the textile unaltered. Using an iron, the film is then heat-transferred onto the textile. We recommend this fabrication approach for tight-fitting garments (e.g., body suits, sports wear), which ensure tight contact of the electrode with the skin.

Ultra-rapid fabrication with PET film: A last approach supports very rapid fabrication, while sacrificing thin and elastic properties. This can be an acceptable trade-off for low-fidelity prototypes during early design stages. The technique uses sinter-free silver-nanoparticle ink, avoiding the need to thermally cure samples, as introduced in [35]. We print on PET film $(\sim 250 \mu \mathrm{m}, 2.5 \mathrm{GPa})$. For electrically insulating conductive traces from the skin, we cover them using transparent scotch tape $(\sim 50 \mu \mathrm{m})$, while leaving the printed electrodes exposed. The printed sheet is adhered to skin using SSA.

\section{Hardware and Interfacing}

We used-off-the shelf commercially available prototyping hardware for controlling our sensors. Olimex EMG/ECG Arduino shields [62] were used for EMG sensing. However, we can anecdotally report that our sensors worked with EMG boards from other manufacturers as well (Seeed Studio,
Groove EMG detector [66]). Sparkfun single lead hardware monitor [63] was used for heart rate/ECG signal acquisition. For EDA monitoring, we used Grove GSR sensor [67]. Data is transmitted wirelessly from the Arduino to a laptop computer using Bluetooth low energy. A python script reads the data and offers a web server streaming interface. While our overall hardware setup can be miniaturized using a custom PCB, our goal was to ensure that the PhysioSkin overlays work with off-the-shelf hardware which is easily accessible.

Connections between the sensor patch and the controller hardware are realized using FPC connectors, to ensure a slim and compact design. The FPC connector is bonded to the printed circuit using conductive z-axis tape (Adafruit). Alternatively, connections can be realized using simple copper tape in prototypes that use only few electrodes.

\section{Integrating User Interface Controls}

To offer options for user input, capacitive touch sensors can be integrated in the designs presented above, without adding to the complexity of fabrication. Electrodes for capacitive sensing are printed on the same layer as physiological sensing electrodes. Contrary to the latter, capacitive electrodes must be insulated from the skin. We achieve this using the same insulation strategy as used for conductive wires. Touch sensing is implemented using self-capacitance sensing with a commercial touch controller (MPR121, NXP Semiconductors). To ensure that the user does not accidentally press the bio-sensing electrodes, the touch electrodes need to be placed $4-5 \mathrm{~cm}$ away from the bio-sensing electrodes. This also reduces the interference between the physiological sensing electrodes and touch electrodes during a touch event.

\section{ACCURACY OF ELECTRO-PHYSIOLOGICAL SENSING}

To understand how well each of these substrate materials monitor bio-signals, we conducted a detailed technical evaluation, with commercial gel-based electrodes as baseline.

\section{Method}

Fabrication approach: We realized one device for each of the fabrication approaches: Tattoo decal paper, PVP, PDMS, textile, PET. The device was bonded to the participant's skin using the respective type of adhesive described above. For the baseline measurements, we used commercial gel-based electrodes (H124SG Covidien).

Sensing modalities: We tested all three modalities: sEMG, ECG and EDA. For sEMG, the electrodes were placed on the Flexor Carpi Radialis muscle of the dominant arm. We chose this muscle since it aids in the wrist movement (flexion) [24]. ECG was measured with electrodes on the chest following the Einthoven's triangle schematic [19]. The EDA electrodes were placed on the thenar and hypo thenar eminence of the dominant hand, since this region has high density of sweat glands [23]. For each combination of modality and substrate, we fabricated separate devices. A device contained 3 electrodes for EMG and ECG sensing and 2 electrodes for EDA. The two measuring electrodes for EMG were on the muscle line, while the third reference electrode was placed on the posterior side of forearm. 


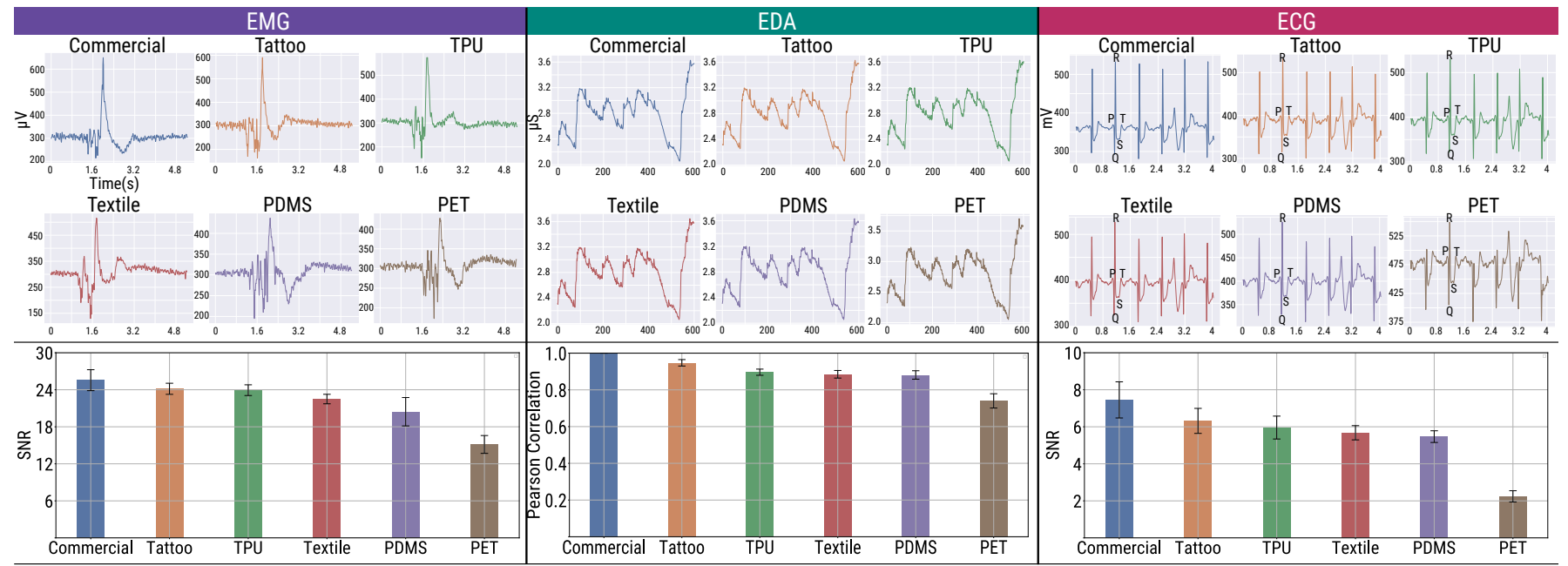

Figure 4. Comparison of SNR and Pearson correlation for EMG, ECG and ED signals for all the substrate conditions. The PQRST waveform is labelled for ECG signals. Error bars indicate standard deviation.

Task: The participants were seated in a comfortable position throughout the entire experiment. For EMG signal acquisition, the participant was asked to perform wrist flexion movement (bending the hand at wrist such that the palm faces the arm) in a comfortable manner, like in prior work [59]. The movement was repeated five times. For EDA and ECG signal acquisition, the participant was at rest, with the hands on a table, while a desktop computer logged the data for 300 seconds. For EDA, the participant underwent a Stroop Color Test [65, 68]. This test has been used in prior work for assessing EDA response. In brief, cognitive stimuli are presented to the subject through the use of words of different colors which are either conflicting (word and color of text are different, i.e., "blue" is written in green color) and non-conflicting (word and color of text are the same). The participant is required to state the color of the word and not read the text. The task consisted of three cycles of $1 \mathrm{~min}$. rest period followed by a $5 \mathrm{~s}$ long Stroop test. This was followed by a final $1 \mathrm{~min}$. rest period.

We recruited 8 participants ( $3 \mathrm{f}$, mean: $28.5 \mathrm{y}$ ). The experiment took 90-120 minutes per participant. The order of FABRICATION APPROACH and SENSING MODALITIES were counterbalanced. The data for each of the modalities was sampled at $250 \mathrm{~Hz}$. Overall we had 8 (PARTICIPANTS) $\times 6$ $($ FABRICATION APPROACH $) \times 3($ SENSING MODALITIES $)=$ 144 sets of measurements.

\section{Analysis}

For EMG signals, we calculated the signal-to-noise ratio (SNR) using a double-threshold detector as stated in prior work [4]:

$$
S N R=10 * \log \left(\frac{\sigma_{s}^{2}}{\sigma_{n}^{2}}-1\right)
$$

where $\sigma_{s}^{2}$ and $\sigma_{n}^{2}$ are the variances of the ON and OFF states, respectively. The $\mathrm{ON}$ state refers to the window where the muscle activity has happened while the OFF state refers to the window where there was no activity. The signal-to-noise ratio for ECG can be calculated as follows [20]:

$$
S N R=\frac{(Q R S) E C G_{p-p}}{(T-P) \text { noise }_{p-p}}
$$

where $E C G_{p-p}$ is peak-to-peak ECG QRS amplitude and noise $_{p-p}$ is peak-to-peak noise amplitude from T-P interval.

For the EDA response, we calculated the Pearson correlation coefficient of each fabrication approach with respect to the baseline condition, based on prior work [27].

\section{Results}

The results are depicted in Figure 4. Overall, our results show that all fabrication approaches realized devices that can reliably capture bio-signals, with tattoo paper substrates performing the best of all fabrication approaches for all modalities. This can be explained by the fact that it has the lowest flexural rigidity of all materials used $\left(\sim 10^{-9} \mathrm{Nm}\right)$.

It is interesting to note that all devices can accurately capture EMG signals. The minimum required SNR for obtaining good EMG measurements was reported to be $20 \mathrm{~dB}$ [10]. All our devices achieve SNRs that are considerably higher. PET is lowest (mean:15.36, SD =1.81), while Tattoo and TPU come close to commercial wet-gel electrodes. This is impressive considering our devices use dry electrodes.

For EDA signals, the tattoo substrate achieves a high correlation and lowest deviation (mean: 0.95, SD: 0.01), again coming close to commercial wet-gel electrodes. TPU, PDMS and Textile follow with means close to 0.9 , while PET shows the least good result (mean: 0.76, sd=0.03).

For ECG signals, the mean average SNR for commercial wetgel electrodes was $7.45 \mathrm{~dB}$ while tattoo based electrodes had a mean SNR of $6.31 \mathrm{~dB}$. Figure 4(c) shows smoothened the ECG signal after applying Hanning window $(\mathrm{n}=11)$. This result is comparable to prior work contributed in the materials community [21]. This suggests that PhysioSkin electrodes can produce meaningful ECG recordings. TPU, Textile and PDMS follow shortly after, with mean average SNRs between 5.5 and $5.8 \mathrm{~dB}$. As can be seen in Figure 4(e), the captured 
signal allows to clearly identify heart rate variability. PET has a considerably lower SNR. As evidenced in the plot, the signal cannot be accurately captured with PET. It is to be noted that all these measurements, including those taken with commercial wet-gel electrodes, are not suitable for clinical recordings, since the minimum required SNR for clinical ECG recordings is $20 \mathrm{~dB}$ [30]. This would require clinical-grade electrode placement and measuring equipment, which is outside the scope of this work.

\section{EXAMPLE APPLICATIONS}

\section{Fitness Tracking Sportswear}

To demonstrate rapid integration of multi-modal sensing in textiles, we implemented a custom sports vest that can track muscle movements and heart rate during exercising (see Fig. 1 (c) and video figure). It uses embedded, conformal textile electrodes and circuitry that is printed and iron-transferred using the method presented above. Locations on the vest were selected such that the electrodes can have good electrical contact with the body. We chose two muscles for EMG monitoring: Biceps Brachii and the anterior part of the Deltoid muscle. The electrodes were placed on based on the recommendations from prior work [29]. Three electrodes for ECG monitoring were placed near the chest. They are connected to an Arduino using standard copper cables. Once the digital design was made, the overall fabrication took approximately 15-20 minutes.

\section{Interactive Heart Rate Sensing Tattoo}

To demonstrate ultra-thin form factors and the ease of integrating input controls, we designed and fabricated a temporary tattoo that can monitor the heart rate activity (Fig. 1 (d) and video figure). It further offers two embedded touch sensors for user input. One button is used for emotional communication purposes, allowing to send one's own live heart beat to a remote loved one. A second button offers privacy options, for turning the sensor on or off. Fabrication took approximately 25-30 minutes (including $\sim 20 \mathrm{mins}$ of heat curing).

\section{Arousal Logging in Virtual Reality Interaction}

Prior work [9] suggested using ECG and EDA to sense emotional arousal and identify the magnitude of the emotional response in immersive VR environments. To realize this approach in a skin-conformal form factor, we implemented a PDMS-based device which can track ECG and the EDA on the forearm. A PDMS based device was fabricated based on the method described previously. The entire procedure took approximately $30-35$ minutes. In our application, we developed a $360^{\circ}$ video viewer which $\log$ s the ECG and EDA data while participants are watching the video. This could be utilized for analysing the arousal patterns.

\section{DISCUSSION, LIMITATIONS AND FUTURE WORK}

EMG Signal Interpretation: The SNR gives a direct correlation with how well the electrodes can pick up muscle activity. From practicality aspect, prior work in biomedical engineering has recommended $[38,10]$ that a SNR $>20 \mathrm{~dB}$ is recommended for detecting precise muscle activations while machine-learning based techniques need to be utilized for signals with lower SNRs ( $>8 \mathrm{~dB}$ ) [38]. These findings have been confirmed for hand gesture classification, showing a $96 \%$ accuracy with 20dB SNR for 7 gestures (1 rest and 6 gestures) using only 4 features[52]. The much higher SNRs identified in our evaluation for all substrate materials (except PET) show that the EMG signals carry enough information for reliable use, e.g., in gesture recognition.

Body Locations: The quality of the signals is dependent on the body locations. In our applications examples, we have deployed the sensors at different body locations. However, the location should be chosen based on the quality of the desired signal. For example, forearm and wrist are not most ideal locations for ECG monitoring which results in a noisy ECG signal, however the heart rate variability can still be detected from the signal due to the QRS peak. For clean ECG signal with distinguishable $\mathrm{PQRST}$ wave, we recommend placing the electrodes near the chest, as in our textile application case.

No clinical grade monitoring: We use hardware from commercial rapid prototyping kits for acquiring bio-signals, rather than clinical grade hardware and materials. Our approach should not be used for clinical-grade monitoring. However, our sensors can be useful for interface designers and hobbyists for quickly prototyping custom physiological sensing solutions for entertainment computing, gesture sensing, or fitness tracking. Additionally, switching to medical grade PDMS, can enable further designs and improve biocompatibility. Future work could address replacement of plasma treatment since it can alter the materials properties and is not easily available.

Scalability: The scalablity of our approach depends on the number of analog pins on the microcontroller and the size available for electrodes on the patch. We used a maximum of 5 channels and an A4-size printer.

Durability: All our substrates (except Tattoo) support usage for multiple times. If the SSA adhesive is used, the patches can be easily applied and re-applied without causing pain or remove of body hairs. SSA is water-proof and can provide good adhesion for long periods of time. Of note, the patches used during our evaluation remained functional even after multiple days and repeated use on multiple users. We can anecdotally report that the textile sensors can withstand multiple washing cycles; a formal study is left for future work.

Extending to other Modalities: Our results show that the electrodes can capture bio-signals when in contact with the body. We therefore believe that our approach should be scalable to further electro-physiological modalities e.g. EOG and EEG, which should be investigated in future work.

\section{CONCLUSION}

In this paper we presented a digital fabrication approach for electro-physiological sensors. With a systematic exploration of materials, functional inks, and adhesives, we demonstrated that custom physiological sensors can be rapidly realized. Informed from the literature we presented a set of design recommendations that can guide designers to realize functional physiological sensing patches. We contributed a comprehensive evaluation across various material substrates, which shows that PhysioSkin devices can capture good-quality bio-signals, and demonstrate working implementations. 


\section{ACKNOWLEDGMENTS}

This project received funding from the European Research Council (ERC) under the European Union's Horizon 2020 research and innovation programme (grant agreement No. 714797 ERC Starting Grant InteractiveSkin). The authors would like to thank Angela Rutz for her assistance in soft-skin adhesive sample preparation and Muhammad Hamid for his help with the video figure.

\section{REFERENCES}

[1] 2020. Apple Watch Series 5 ECG/Heart Rate. https://www . apple. com/apple-watch-series-5/health/. (2020). Accessed: 2020-01-08.

[2] 2020. Hexoskin Health Sensors and AI. http://https://www.hexoskin. com/. (2020). Last Accessed: 2020-01-08.

[3] Gizem Acar, Ozberk Ozturk, and Murat Kaya Yapici. 2018. Wearable Graphene Nanotextile Embedded Smart Armband for Cardiac Monitoring. In 2018 IEEE SENSORS. IEEE, 1-4.

[4] Valentina Agostini and Marco Knaflitz. 2011. An algorithm for the estimation of the signal-to-noise ratio in surface myoelectric signals generated during cyclic movements. IEEE Transactions on Biomedical Engineering 59, 1 (2011), 219-225.

[5] Christoph Amma, Thomas Krings, Jonas Böer, and Tanja Schultz. 2015. Advancing muscle-computer interfaces with high-density electromyography. In Proceedings of the 33rd Annual ACM Conference on Human Factors in Computing Systems. ACM, 929-938.

[6] AS Anusha, SP Preejith, Tony J Akl, Jayaraj Joseph, and Mohanasankar Sivaprakasam. 2018. Dry Electrode Optimization for Wrist-based Electrodermal Activity Monitoring. In 2018 IEEE International Symposium on Medical Measurements and Applications (MeMeA). IEEE, 1-6.

[7] Marco Barbero, Roberto Merletti, and Alberto Rainoldi. 2012. Atlas of muscle innervation zones: understanding surface electromyography and its applications. Springer Science \& Business Media.

[8] Sharon Baurley, Philippa Brock, Erik Geelhoed, and Andrew Moore. 2007. Communication-Wear: user feedback as part of a co-design process. In International Workshop on Haptic and Audio Interaction Design. Springer, 56-68.

[9] Guillermo Bernal, Tao Yang, Abhinandan Jain, and Pattie Maes. 2018. PhysioHMD: A Conformable, Modular Toolkit for Collecting Physiological Data from Head-mounted Displays. In Proceedings of the 2018 ACM International Symposium on Wearable Computers (ISWC '18). ACM, New York, NY, USA, 160-167. DOI : http://dx.doi .org/10.1145/3267242.3267268

[10] Paolo Bonato, Tommaso D’Alessio, and Marco Knaflitz. 1998. A statistical method for the measurement of muscle activation intervals from surface myoelectric signal during gait. IEEE Transactions on biomedical engineering 45, 3 (1998), 287-299.

[11] Wolfram Boucsein. 2012. Electrodermal activity. Springer Science \& Business Media.

[12] Varun Perumal C and Daniel Wigdor. 2015. Printem: Instant Printed Circuit Boards with Standard Office Printers Inks. In Proceedings of the 28th Annual ACM Symposium on User Interface Software Technology (UIST '15). Association for Computing Machinery, New York, NY, USA, 243-251. DOI : http://dx.doi.org/10.1145/2807442.2807511

[13] Andrew Carek and Christian Holz. 2018. Naptics: Convenient and Continuous Blood Pressure Monitoring during Sleep. Proceedings of the ACM on Interactive, Mobile, Wearable and Ubiquitous Technologies 2, 3 (2018), 96.

[14] C. Cochrane, C. Hertleer, and A. Schwarz-Pfeiffer. 2016. Smart textiles in health: An overview. In Smart Textiles and their Applications, Vladan Koncar (Ed.). Woodhead Publishing, Oxford, 9 - 32. DOI :

http://dx.doi.org/https:

//doi.org/10.1016/B978-0-08-100574-3.00002-3

[15] Mary Boudreau Conover. 2002. Understanding electrocardiography. Elsevier Health Sciences.

[16] Artem Dementyev, Javier Hernandez, Inrak Choi, Sean Follmer, and Joseph Paradiso. 2018. Epidermal Robots: Wearable Sensors That Climb on the Skin. Proceedings of the ACM on Interactive, Mobile, Wearable and Ubiquitous Technologies 2, 3 (2018), 102.

[17] Artem Dementyev and Christian Holz. 2017. DualBlink: a wearable device to continuously detect, track, and actuate blinking for alleviating dry eyes and computer vision syndrome. Proceedings of the ACM on Interactive, Mobile, Wearable and Ubiquitous Technologies 1, 1 (2017), 1.

[18] Artem Dementyev, Hsin-Liu Cindy Kao, and Joseph A Paradiso. 2015. Sensortape: Modular and programmable 3d-aware dense sensor network on a tape. In Proceedings of the 28th Annual ACM Symposium on User Interface Software \& Technology. ACM, 649-658.

[19] Willem Einthoven, G Fahr, and A De Waart. 1913. "U about the direction and the manifest size of the potential fluctuations in the human heart and about the influence of the heart situation on the shape of the electrocardiogram. Pflügler's Archive European Journal of Physiology 150, 6 (1913), 275-315.

[20] Omar J Escalona, Louise McFrederick, Maira Borges, Pedro Linares, Ricardo Villegas, Gilberto I Perpiñan, James McLaughlin, and David McEneaney. 2017. Wrist and arm body surface bipolar ECG leads signal and sensor study for long-term rhythm monitoring. In 2017 Computing in Cardiology (CinC). IEEE, 1-4. 
[21] Laura M Ferrari, Sudha Sudha, Sergio Tarantino, Roberto Esposti, Francesco Bolzoni, Paolo Cavallari, Christian Cipriani, Virgilio Mattoli, and Francesco Greco. 2018. Ultraconformable temporary tattoo electrodes for electrophysiology. Advanced Science 5, 3 (2018), 1700771.

[22] T Finni, Min Hu, P Kettunen, T Vilavuo, and S Cheng. 2007. Measurement of EMG activity with textile electrodes embedded into clothing. Physiological measurement 28, 11 (2007), 1405.

[23] Don C Fowles, Margaret J Christie, Robert Edelberg, William W Grings, David T Lykken, and Peter H Venables. 1981. Publication recommendations for electrodermal measurements. Psychophysiology 18, 3 (1981), 232-239.

[24] Hossein Ghapanchizadeh, Siti A Ahmad, and Asnor Juraiza Ishak. 2015. Recommended surface EMG electrode position for wrist extension and flexion. In 2015 IEEE Student Symposium in Biomedical Engineering \& Sciences (ISSBES). IEEE, 108-112.

[25] Stephen Gilroy, Julie Porteous, Fred Charles, and Marc Cavazza. 2012. PINTER: Interactive Storytelling with Physiological Input. In Proceedings of the 2012 ACM International Conference on Intelligent User Interfaces (IUI '12). ACM, New York, NY, USA, 333-334. DOI : http://dx.doi.org/10.1145/2166966.2167039

[26] Daniel Groeger and Jürgen Steimle. 2019. LASEC: Instant Fabrication of Stretchable Circuits Using a Laser Cutter. In Proceedings of the 2019 CHI Conference on Human Factors in Computing Systems (CHI '19). ACM, New York, NY, USA, Article 699, 14 pages. DOI: http://dx.doi.org/10.1145/3290605.3300929

[27] Peter A Haddad, Amir Servati, Saeid Soltanian, Frank Ko, and Peyman Servati. 2017. Effects of flexible dry electrode design on electrodermal activity stimulus response detection. IEEE Transactions on Biomedical Engineering 64, 12 (2017), 2979-2987.

[28] Manne Hannula, H Hinkula, and J Jauhiainen. 2008. Development and evaluation of one arm electrode based ECG measurement system. In 14th Nordic-Baltic Conference on Biomedical Engineering and Medical Physics. Springer, 234-237.

[29] Hermie J Hermens, Bart Freriks, Catherine Disselhorst-Klug, and Günter Rau. 2000. Development of recommendations for SEMG sensors and sensor placement procedures. Journal of Electromyography and Kinesiology 10, 5 (oct 2000), 361-374. DOI : http://dx.doi.org/10.1016/S1050-6411(00)00027-4

[30] Rafael E Herrera, James T Cain, EG Cape, and Gerard J Boyle. 1996. A high resolution ECG tool for detection of atrial and ventricular late potentials. In Computers in Cardiology 1996. IEEE, 629-632.
[31] Christian Holz and Edward J Wang. 2017. Glabella: Continuously sensing blood pressure behavior using an unobtrusive wearable device. Proceedings of the ACM on Interactive, Mobile, Wearable and Ubiquitous Technologies 1, 3 (2017), 58.

[32] Xian Huang, Yuhao Liu, Kaile Chen, Woo-Jung Shin, Ching-Jui Lu, Gil-Woo Kong, Dwipayan Patnaik, Sang-Heon Lee, Jonathan Fajardo Cortes, and John A Rogers. 2014. Stretchable, wireless sensors and functional substrates for epidermal characterization of sweat. Small 10, 15 (2014), 3083-3090.

[33] Phil Jevon. 2010. Procedure for recording a standard 12-lead electrocardiogram. British Journal of Nursing 19, 10 (2010), 649-651.

[34] Hsin-Liu Cindy Kao, Christian Holz, Asta Roseway, Andres Calvo, and Chris Schmandt. 2016. DuoSkin: rapidly prototyping on-skin user interfaces using skin-friendly materials. In Proceedings of the 2016 ACM International Symposium on Wearable Computers. ACM, 16-23.

[35] Yoshihiro Kawahara, Steve Hodges, Benjamin S. Cook, Cheng Zhang, and Gregory D. Abowd. 2013. Instant Inkjet Circuits: Lab-based Inkjet Printing to Support Rapid Prototyping of UbiComp Devices. In Proceedings of the 2013 ACM International Joint Conference on Pervasive and Ubiquitous Computing (UbiComp '13). ACM, New York, NY, USA, 363-372. DOI : http://dx.doi.org/10.1145/2493432.2493486

[36] Arshad Khan, Joan Sol Roo, Tobias Kraus, and Jürgen Steimle. 2011. Soft Inkjet Circuits: Rapid Multi-Material Fabrication of Soft Circuits using a Commodity Inkjet Printer. In Proceedings of the 32nd Annual ACM Symposium on User Interface Software and Technology (UIST' 19). ACM, New York, NY, USA, 10.

[37] Jeonghyun Kim, Philipp Gutruf, Antonio M Chiarelli, Seung Yun Heo, Kyoungyeon Cho, Zhaoqian Xie, Anthony Banks, Seungyoung Han, Kyung-In Jang, Jung Woo Lee, and others. 2017. Oximetry: Miniaturized Battery-Free Wireless Systems for Wearable Pulse Oximetry (Adv. Funct. Mater. 1/2017). Advanced Functional Materials 27, 1 (2017).

[38] Jie Liu, Dongwen Ying, William Z Rymer, and Ping Zhou. 2015. Robust muscle activity onset detection using an unsupervised electromyogram learning framework. PloS one 10, 6 (2015), e0127990.

[39] Joanne Lo, Doris Jung Lin Lee, Nathan Wong, David Bui, and Eric Paulos. 2016. Skintillates: Designing and creating epidermal interactions. In Proceedings of the 2016 ACM Conference on Designing Interactive Systems. ACM, 853-864.

[40] Johan Löfhede, Fernando Seoane, and Magnus Thordstein. 2012. Textile electrodes for EEG recording-A pilot study. Sensors 12, 12 (2012), 16907-16919. 
[41] Eric Markvicka, Guanyun Wang, Yi-Chin Lee, Gierad Laput, Carmel Majidi, and Lining Yao. 2019. ElectroDermis: Fully Untethered, Stretchable, and Highly-Customizable Electronic Bandages. In Proceedings of the 2019 CHI Conference on Human Factors in Computing Systems (CHI '19). Association for Computing Machinery, New York, NY, USA, Article Paper 632, 10 pages. DOI :

http://dx.doi.org/10.1145/3290605.3300862

[42] David A Mellis, Sam Jacoby, Leah Buechley, Hannah Perner-Wilson, and Jie Qi. 2013. Microcontrollers as material: crafting circuits with paper, conductive ink, electronic components, and an untoolkit. In Proceedings of the 7th International Conference on Tangible, Embedded and Embodied Interaction. ACM, 83-90.

[43] Roberto Merletti, L Lo Conte, Elena Avignone, and Piero Guglielminotti. 1999. Modeling of surface myoelectric signals. I. Model implementation. IEEE transactions on biomedical engineering 46, 7 (1999), 810-820.

[44] Roberto Merletti, Philip A Parker, and Philip J Parker. 2004. Electromyography: physiology, engineering, and non-invasive applications. Vol. 11. John Wiley \& Sons.

[45] Steven Nagels, Raf Ramakers, Kris Luyten, and Wim Deferme. 2018. Silicone Devices: A Scalable DIY Approach for Fabricating Self-Contained Multi-Layered Soft Circuits Using Microfluidics. In Proceedings of the 2018 CHI Conference on Human Factors in Computing Systems. ACM, 188.

[46] Aditya Shekhar Nittala, Klaus Kruttwig, Jaeyeon Lee, Roland Bennewitz, Eduard Arzt, and Jürgen Steimle. 2019. Like a Second Skin: Understanding How Epidermal Devices Affect Human Tactile Perception. In Proceedings of the 2019 CHI Conference on Human Factors in Computing Systems. ACM, 380.

[47] Aditya Shekhar Nittala, Anusha Withana, Narjes Pourjafarian, and Jürgen Steimle. 2018. Multi-Touch Skin: A Thin and Flexible Multi-Touch Sensor for On-Skin Input. In Proceedings of the 2018 CHI Conference on Human Factors in Computing Systems. ACM, 33.

[48] Simon Olberding, Nan-Wei Gong, John Tiab, Joseph A. Paradiso, and Jürgen Steimle. 2013. A Cuttable Multi-touch Sensor. In Proceedings of the 26th Annual ACM Symposium on User Interface Software and Technology (UIST '13). ACM, New York, NY, USA, 245-254. DOI :

http://dx.doi.org/10.1145/2501988.2502048

[49] Patrick Parzer, Florian Perteneder, Kathrin Probst, Christian Rendl, Joanne Leong, Sarah Schuetz, Anita Vogl, Reinhard Schwoediauer, Martin Kaltenbrunner, Siegfried Bauer, and Michael Haller. 2018. RESi: A Highly Flexible, Pressure-Sensitive, Imperceptible Textile Interface Based on Resistive Yarns. In Proceedings of the 31st Annual ACM Symposium on
User Interface Software and Technology (UIST '18). ACM, New York, NY, USA, 745-756. DOI : http://dx. doi .org/10.1145/3242587.3242664

[50] Patrick Parzer, Adwait Sharma, Anita Vogl, Jürgen Steimle, Alex Olwal, and Michael Haller. 2017. SmartSleeve: real-time sensing of surface and deformation gestures on flexible, interactive textiles, using a hybrid gesture detection pipeline. In Proceedings of the 30th Annual ACM Symposium on User Interface Software and Technology. ACM, 565-577.

[51] Shyamal Patel, Hyung Park, Paolo Bonato, Leighton Chan, and Mary Rodgers. 2012. A review of wearable sensors and systems with application in rehabilitation. Journal of neuroengineering and rehabilitation 9, 1 (2012), 21.

[52] Angkoon Phinyomark, Chusak Limsakul, and Pornchai Phukpattaranont. 2009. A novel feature extraction for robust EMG pattern recognition. arXiv preprint arXiv:0912.3973 (2009).

[53] Jie Qi and Leah Buechley. 2014. Sketching in circuits: designing and building electronics on paper. In Proceedings of the SIGCHI Conference on Human Factors in Computing Systems. ACM, 1713-1722.

[54] Raf Ramakers, Kashyap Todi, and Kris Luyten. 2015. PaperPulse: An Integrated Approach for Embedding Electronics in Paper Designs. In Proceedings of the 33rd Annual ACM Conference on Human Factors in Computing Systems (CHI '15). ACM, New York, NY, USA, 2457-2466. DOI:

http://dx.doi.org/10.1145/2702123.2702487

[55] Linda Rattfält. 2013. Smartware electrodes for ECG measurements -Design, evaluation and signal processing. Number 1546.

[56] Linda Rattfält, Fredrik Björefors, David Nilsson, Xin Wang, Petronella Norberg, Per Ask, and Linda Rattfalt@liu Se. 2013. Properties of screen printed electrocardiography smartware electrodes investigated in an electro-chemical cell. Technical Report. DOI :

http://dx.doi .org/10.1186/1475-925X-12-64

[57] Bersain A Reyes, Hugo F Posada-Quintero, Justin R Bales, Amanda L Clement, George D Pins, Albert Swiston, Jarno Riistama, John P Florian, Barbara Shykoff, Michael Qin, and others. 2014. Novel electrodes for underwater ECG monitoring. IEEE Transactions on Biomedical Engineering 61, 6 (2014), 1863-1876.

[58] Shigeru Sakurazawa, Naofumi Yoshida, and Nagisa Munekata. 2004. Entertainment Feature of a Game Using Skin Conductance Response. In Proceedings of the 2004 ACM SIGCHI International Conference on Advances in Computer Entertainment Technology (ACE '04). ACM, New York, NY, USA, 181-186. DOI: http://dx.doi.org/10.1145/1067343.1067365 
[59] T Scott Saponas, Desney S Tan, Dan Morris, Ravin Balakrishnan, Jim Turner, and James A Landay. 2009. Enabling always-available input with muscle-computer interfaces. In Proceedings of the 22nd annual ACM symposium on User interface software and technology. ACM, 167-176.

[60] T Scott Saponas, Desney S Tan, Dan Morris, Jim Turner, and James A Landay. 2010. Making muscle-computer interfaces more practical. In Proceedings of the SIGCHI Conference on Human Factors in Computing Systems. ACM, 851-854.

[61] Valkyrie Savage, Xiaohan Zhang, and Björn Hartmann. 2012. Midas: fabricating custom capacitive touch sensors to prototype interactive objects. In Proceedings of the 25th annual ACM symposium on User interface software and technology. ACM, 579-588.

[62] Olimex EKG-EMG Shield. 2020. Open Source EMG-ECG Shields. (2020).

https://www. olimex. com/Products/Duino/Shields/ SHIELD-EKG-EMG/open-source-hardware Last Accessed: 2020-01-08.

[63] Sparkfun. 2020. SparkFun Single Lead Heart Rate Monitor. https://learn. sparkfun. com/tutorials/ ad8232-heart-rate-monitor-hookup-guide/all. (2020). Last Accessed: 2020-01-08.

[64] Jürgen Steimle, Joanna Bergstrom-Lehtovirta, Martin Weigel, Aditya Shekhar Nittala, Sebastian Boring, Alex Olwal, and Kasper Hornbæk. 2017. On-skin interaction using body landmarks. Computer 50, 10 (2017), 19-27.

[65] J Ridley Stroop. 1992. Studies of interference in serial verbal reactions. Journal of Experimental Psychology: General 121, 1 (1992), 15.

[66] Seeed Studio. 2020a. Groove EMG Detector. http://wiki.seeedstudio.com/Grove-EMG_Detector/. (2020). Last Accessed: 2020-01-08.

[67] Seeed Studio. 2020b. Groove GSR Sensor. http://wiki.seeedstudio.com/Grove-GSR_Sensor/. (2020). Last Accessed: 2020-01-08.

[68] Miroslav Svetlak, Petr Bob, Michal Cernik, and Miloslav Kukleta. 2010. Electrodermal complexity during the Stroop colour word test. Autonomic Neuroscience 152, 1-2 (2010), 101-107.

[69] Marieke van Dooren, J.J.G. (Gert-Jan) de Vries, and Joris H. Janssen. 2012. Emotional sweating across the body: Comparing 16 different skin conductance measurement locations. Physiology \& Behavior 106, 2 (may 2012), 298-304. DOI:

http://dx.doi.org/10.1016/J.PHYSBEH. 2012.01.020
[70] Chiuan Wang, Hsuan-Ming Yeh, Bryan Wang, Te-Yen Wu, Hsin-Ruey Tsai, Rong-Hao Liang, Yi-Ping Hung, and Mike Y. Chen. 2016. CircuitStack: Supporting Rapid Prototyping and Evolution of Electronic Circuits. In Proceedings of the 29th Annual Symposium on User Interface Software and Technology (UIST '16). ACM, New York, NY, USA, 687-695. DOI:

http://dx.doi.org/10.1145/2984511.2984527

[71] R Chad Webb, Andrew P Bonifas, Alex Behnaz, Yihui Zhang, Ki Jun Yu, Huanyu Cheng, Mingxing Shi, Zuguang Bian, Zhuangjian Liu, Yun-Soung Kim, and others. 2013. Ultrathin conformal devices for precise and continuous thermal characterization of human skin. Nature materials 12, 10 (2013), 938.

[72] Martin Weigel, Tong Lu, Gilles Bailly, Antti Oulasvirta, Carmel Majidi, and Jürgen Steimle. 2015. Iskin: flexible, stretchable and visually customizable on-body touch sensors for mobile computing. In Proceedings of the 33rd Annual ACM Conference on Human Factors in Computing Systems. ACM, 2991-3000.

[73] Martin Weigel, Aditya Shekhar Nittala, Alex Olwal, and Jürgen Steimle. 2017. SkinMarks : Enabling Interaction on Body Landmarks Using Conformal Skin Electronics. In Proceedings of the SIGCHI Conference on Human Factors in Computing Systems (CHI '17). ACM, New York, NY, USA. DOI : http://dx.doi .org/10.1145/3025453. 3025704

[74] Anusha Withana, Daniel Groeger, and Jürgen Steimle. 2018. Tacttoo: A Thin and Feel-Through Tattoo for On-Skin Tactile Output. In The 31st Annual ACM Symposium on User Interface Software and Technology. ACM, 365-378.

[75] PJ Xu, H Zhang, and XM Tao. 2008. Textile-structured electrodes for electrocardiogram. Textile Progress 40, 4 (2008), 183-213.

[76] Hung-Chi Yang, Tsung-Fu Chien, Shang-Hao Liu, and Hsuan-Han Chiang. Study of Single-Arm Electrode for ECG Measurement Using Flexible Print Circuit. (????).

[77] Woon-Hong Yeo, Yun-Soung Kim, Jongwoo Lee, Abid Ameen, Luke Shi, Ming Li, Shuodao Wang, Rui Ma, Sung Hun Jin, Zhan Kang, and others. 2013. Multifunctional epidermal electronics printed directly onto the skin. Advanced Materials 25, 20 (2013), 2773-2778.

[78] P Zipp. 1982. Recommendations for the Standardization of Lead Positions in Surface Electromyography*. Technical Report. 41-54 pages. https://link. springer. com/content/pdf/10.1007/BF00952243.pdf 